\title{
Genome-wide association analysis of 95,549 individuals identifies novel loci and genes influencing optic disc morphology
}

Xikun Han, ${ }^{* \# 1,2}$ Ayub Qassim, ${ }^{\# 3}$ Jiyuan An, ${ }^{1}$ Henry Marshall, ${ }^{3}$ Tiger Zhou, ${ }^{3}$ Jue-Sheng Ong, ${ }^{1}$ Mark Hassall, ${ }^{3}$ Pirro G Hysi, ${ }^{4}$ Paul J Foster, ${ }^{5}$ Peng T Khaw, ${ }^{5}$ David A Mackey, ${ }^{6,7}$ Puya Gharahkhani, ${ }^{1}$ Anthony P Khawaja, ${ }^{5}$ Alex W Hewitt $, 6,8$ Jamie E Craig, ${ }^{3}$ Stuart MacGregor ${ }^{1}$

Affiliations:

1. Statistical Genetics, QIMR Berghofer Medical Research Institute, Brisbane, Australia.

2. School of Medicine, University of Queensland, St Lucia, Brisbane, Australia.

3. Department of Ophthalmology, Flinders University, Flinders Medical Centre, Bedford Park, Australia.

4. Department of Ophthalmology, King's College London, St. Thomas' Hospital, London, UK

5. NIHR Biomedical Research Centre, Moorfields Eye Hospital NHS Foundation Trust and UCL Institute of Ophthalmology, London, UK

6. Menzies Institute for Medical Research, University of Tasmania, Australia.

7. Lions Eye Institute, Centre for Vision Sciences, University of Western Australia, Australia.

8. Centre for Eye Research Australia, University of Melbourne, Australia.

\# Contributed equally

${ }^{*}$ Correspondence:

Xikun Han, Statistical Genetics, QIMR Berghofer Medical Research Institute, Brisbane, Australia.

E-mail: Xikun.Han@qimrberghofer.edu.au

300 Herston Road, Herston, Brisbane, Queensland 4006, Australia 


\begin{abstract}
Optic nerve head morphology is affected by several retinal diseases. We measured the vertical optic disc diameter (DD) of the UK Biobank cohort (UKBB, N=67,040), and performed the largest genome-wide association study (GWAS) of DD to date. We identified 81 loci (66 novel) for vertical DD. We then replicated the novel loci in International Glaucoma Genetic Consortium (IGGC, N=22,504) and EPIC-Norfolk $(\mathrm{N}=6,005)$; in general the concordance in effect sizes was very high (correlation in effect size estimates 0.90$)$, 44 of the 66 novel loci were significant at $\mathrm{P}<0.05$, with 19 remaining significant after Bonferroni correction. We identified another 26 novel loci in the meta-analysis of UKBB and IGGC data. Gene-based analyses identified an additional 57 genes. Human ocular tissue gene expression analysis showed most of the identified genes are enriched in optic nerve head tissue. Some of the identified loci exhibited pleiotropic effects with vertical cup-to-disc ratio, intraocular pressure, glaucoma, and myopia. These results can enhance our understanding of the genetics of optic disc morphology and shed light on the genetic findings for other ophthalmic disorders such as glaucoma and other optic nerve diseases.
\end{abstract}




\section{Introduction}

The optic disc is an oval structure representing the exit point of the retinal ganglion cell axons as they form the optic nerve responsible for transmitting vision to the brain. Anatomically, the optic disc can be divided into the neuroretinal rim where the nerves fibre layer turns outwards away from the retina, and the optic cup, which does not contain any nerve fibre layer and is located more central to the neuroretinal rim. The optic disc is clinically examined using fundoscopy and optical imaging technology (such as the Heidelberg Retinal Tomography [HRT] or the Optical Coherence Tomography [OCT]) for signs of retinal and optic nerve diseases. $(1,2)$ Common metrics of the optic disc morphology include the disc size (measured as the vertical disc diameter [DD] or disc area) and the vertical cup-to-disc ratio (VCDR). Primary open angle glaucoma (POAG) is an optic neuropathy characterised by an accelerated loss of the retinal ganglion layer and is a leading cause of blindness globally.(3) The loss of the retinal ganglion cells manifests as an enlarged optic cup and VCDR and is considered a hallmark of POAG.(4)

A better understanding of factors that influence optic disc size is of high clinical relevance. Optic disc size affects the structural morphology of the optic nerve head and may influence the vulnerability of the nerve fibres.(5) Small optic nerves are associated with disorders such as optic nerve hypoplasia and increased risk of Non-arteritic Anterior Ischaemic Optic Neuropathy and Optic Disc Drusen. $(6,7)$ There is a strong correlation between the optic disc size and the VCDR (clinically and genetically) and this should be taken into account in funduscopic examination.(8) Adjusting optic disc parameters such as VCDR for disc size improves their diagnostic power and clinical utility for glaucoma assessment.(9) For example, adjusting the VCDR to DD improves its sensitivity of identifying eyes with perimetric glaucoma from $67 \%$ to $76.6 \%$ (at $80 \%$ specificity).(9) Optic disc morphology traits are highly heritable with an estimated heritability of disc size and VCDR of 0.48 - 0.57 from family studies while the single nucleotide polymorphism (SNP) -based heritability are estimated to range from 0.27 to $0.31 .(10,11)$ To date, however, less than 20 genes are shown to be implicated with disc size.(11-16) In this study, we conduct the largest genome-wide association study (GWAS) for disc size (triple the previously studied sample size) to investigate the development mechanisms of the optic nerve and shed light on the genetic understanding for some eye diseases such as POAG and other optic nerve diseases. 


\section{Results}

In the discovery stage, we conducted GWAS on vertical DD in 67,040 UK Biobank (UKBB) samples, then we replicated the novel associated candidate loci in independent cohorts from the International Glaucoma Genetics Consortium (IGGC, N=22,504) and EPIC-Norfolk (N=6,005).

\section{UKBB disc diameter GWAS identifies 66 novel loci}

From the vertical disc diameter GWAS of 67,040 UKBB participants, we identified 91 lead genome-wide significant independent SNPs (81 loci), of which 67 SNPs (66 loci) had not previously been associated with disc diameter (Figure 1A, Supplementary Figure S1 and Table S1). Interestingly, we also identified two genes located in the X chromosome (EFNB1 and ZIC3, Supplementary Table S2), which play an important role in eye development. $(17,18)$ We conducted linkage disequilibrium (LD) score regression and observed no evidence for genomic inflation (intercept=1.05, SE=0.01, Supplementary Figure S2). As previously reported,(11) the genetic correlation between disc diameter and VCDR was very high $\left(\mathrm{rg}=0.50, \mathrm{P}=6.18 \times 10^{-}\right.$

$\left.{ }^{21}\right)$. The genetic correlation between DD and POAG was very small $(\mathrm{rg}=0.01, \mathrm{P}=0.78)$. The strong association between DD and VCDR is due to the physiologically larger vertical cup diameter and optic disc rim area found in larger optic discs.(8) A higher count of optic nerve fibres is found histologically in eyes with larger optic discs,(19) representing the larger neuroretinal rim area seen on fundoscopy. The genetic correlation of disc size between UKBB and IGGC was $0.83\left(\mathrm{P}=1.31 \times 10^{-76}\right)$.

\section{Replication in IGGC and EPIC-Norfolk datasets}

We then replicated the identified lead vertical DD loci in IGGC and EPIC-Norfolk datasets. The correlation in effect size estimates at the lead genome-wide significant SNPs were $0.90\left(\mathrm{P}=2.85 \times 10^{-33}\right.$, Figure 2), indicating the identified disc diameter SNPs from UKBB could be well replicated (Supplementary Table S1). Of the 64 novel loci from autosomal chromosomes, 19 loci could be replicated in IGGC and EPIC-Norfolk after Bonferroni correction $\left(\mathrm{P}=0.05 / 64=7.8 \times 10^{-4}\right.$, Table 1$)$, and 44 loci have nominal association $(\mathrm{P}=0.05$, Supplementary Table S1). The X chromosome GWAS results are not available in the IGGC and EPIC-Norfolk cohorts. Therefore, further studies are needed to confirm the associations of these two X chromosome genes with DD. 


\section{Meta-analysis of UKBB and IGGC}

We subsequently conducted a GWAS meta-analysis to combine UKBB and IGGC disc size datasets, and identified 115 independent genome-wide significant SNPs (from 101 loci, and an additional 26 novel disc size loci, Supplementary Figure S3 and Table S3).

\section{Functional annotation}

We further investigated the potential biological functions of the identified genome-wide significant variants $\left(\mathrm{P}<5 \times 10^{-8}\right)$. Figure $3 \mathrm{~A}$ showed the functional categories of the genome-wide significant variants. We found $0.83 \%$ of the significant variants (77 variants from 39 genes, Supplementary Table S4) located in the exonic regions, and $85 \%$ of the significant variants were intronic or intergenic. A 15-core chromatin state was evaluated for 127 tissue or cell types,(20,21) and we found 5.48\% (510 variants, Figure 3B and Supplementary Table S5) significant variants were active transcription start sites. The RegulomeDB score was used to identify regulatory elements, and Figure 3C indicated that 1.36\% (113 variants, Figure 3C and Supplementary Table S6) significant variants were at least eQTL and transcription factor binding sites. Figure 3D presented the Combined Annotation Dependent Depletion (CADD) score distribution, which is an integrative metric to measure variant deleteriousness. $(22,23)$ The higher the score, the more likely that a SNP is deleteriousness (suggested threshold 12.37),(22) which could be used to prioritize causal variations (Supplementary Table S7).

\section{Gene-based and pathway analysis}

We then conducted a genome-wide gene-based association analysis and identified an additional 57 novel genes (without genome-wide significant SNPs in genes, Supplementary Figure S4 and Table S8). For instance, gene THSD4 was associated with eye tail length and outercanthal width,(24) and genes RNLS, DENNDIA, RASGEF1B, FAM15OB, and NCOA2 were associated with myopia. Tissue expression analysis of GTEx data (V7 30 general tissue types) indicated the gene expression profiles were enriched in nerve tissue (Supplementary Figure S5). Pathway analysis of 10,678 gene sets (MsigDB v6.2, curated gene sets: 4,761, Gene Ontology terms: 5,917) resulted in 29 significant gene sets after Bonferroni correction, which include sensory organ development, tissue development, and morphogenesis (Supplement Table S9). The top pathway is RAMJAUN_APOPTOSIS_BY_TGFB1_VIA_MAPK1_UP, which is a transforming growth factor-beta (TGFbeta) activated signalling pathway, involved in apoptosis and the regulation of cell growth and survival. 


\section{Genetic correlation with other traits}

We estimated the genetic correlation between disc size and 832 traits in LD-Hub database (v1.9.0).(27) We only found significant genetic correlation between disc size and myopia (UKBB data field 6147: Reason for glasses/contact lenses, $\mathrm{rg}=-0.24, \mathrm{P}=5.94 \times 10^{-8}$, Supplementary Table S10) after Bonferroni correction $(0.05 / 832)$.

We also investigated GWAS Catalog(28), a curated collection of published genome-wide association studies, for disc size genome-wide significant SNPs (Supplementary Table S11). Our results showed some of the lead disc size loci had pleiotropy effects. For instance, lead SNPs in genes CDC42BPA and ANKRD55 were associated with macular thickness, and lead SNPs in ANKRD55, PRSS56, KCNQ5, NPLOC4, and BMP4 were related to myopia.

\section{Gene expression in human ocular tissues}

We also investigated the expression profile of the genes nearest to the identified SNPs in ocular tissue: optic nerve head, optic nerve, retina, trabecular meshwork, iris, ciliary body, sclera, cornea, as well as foetal retinal tissue (Supplementary Table S12, Table S13, Supplementary Figure S6 and S7).(29) The majority (94/106, 89\%) displayed differential expression in the optic nerve head relative to all other ocular tissue (Supplementary Table S14). BCAS3 (Microtubule Associated Cell Migration Factor), DHRS7 (Dehydrogenase/Reductase 7) and NPLOC4 (Nuclear Protein Localization Protein 4 Homolog) were the most significantly differentially expressed genes in the optic nerve head and were all novel discoveries. The SNP rs12147505 in DHRS7 with no linkage disequilibrium with rs34935520 (in SIX6, $\left.\mathrm{R}^{2}<0.001\right)(11)$, had the highest magnitude of effect on disc size; it is a protein coding gene functioning as a catalyst in oxidation and reduction of a wide range of substrates.(30) It is expressed in all ocular tissues, with highest expression in the corneal stroma followed by the optic nerve head, and suggested to be a risk locus for POAG.(31)

\section{eQTL and transcriptome-wide association analysis}

We looked up the lead DD genome-wide significant SNPs in retina from the Eye Genotype Expression (EyeGEx) database to identify expression quantitative trait loci (eQTL).(32) We identified 38 SNP-gene pairs (cis-eQTLs) after gene-level multiple testing correction across the genome (Supplementary Table S15). We also conducted summary data-based Mendelian randomization (SMR) and heterogeneity in dependent 
instruments (HEIDI) analysis to test the effects of genetic variants on disc size that is mediated by gene expression levels.(33) From SMR approach, we identified 15 genes after multiple testing correction $\left(\mathrm{P}_{\mathrm{SMR}}<\right.$ $0.05 / 5592=8.94 \times 10^{-6}$, Supplementary Table S16). The HEIDI tests $\left(\mathrm{P}_{\text {HEIDI }} \geq 0.05\right)$ suggested that genes TANC2 and CTD-2292P10.4 are associated with DD via the underlying GWAS hits.

\section{Discussion}

We conducted the largest optic disc size GWAS to date and identified 101 loci including 159 genes using manual grading of the UKBB fundus photographs. We identified for the first time two genes located in the $\mathrm{X}$ chromosome (EFNB1 and ZIC3) associated with vertical disc diameter. Our results indicate that sensory organ development, tissue development, and morphogenesis are involved in the biological pathway for optic disc morphology. The discovery of genes and pathways involved in optic disc morphology is important in the understanding of the genetic architecture and development mechanisms of optic nerve head and would increase our knowledge of diseases related to this - POAG and other optic nerve diseases.

The identified optic disc size genes have important functions. For instance, the top two novel replicated genes are VANGL1 and CENPW. VANGL Planar Cell Polarity Protein 1 (VANGL1) regulates the establishment of planar cell polarity, which plays a key role in tissue morphogenesis, embryonic development, and the development of eye tissues.(34-36) CENPW encodes Centromere Protein W, which is related to cell cycle, mitotic state, and chromosome maintenance.(37) The lead SNP rs2152876 in CENPW exhibits a pleiotropic effect, as its proxy SNPs $\left(\mathrm{R}^{2}>0.8\right)$ are associated with intraocular pressure(38), height(39), hip circumference(40), and the age onset of menarche(41). The encoded protein by STRA6 acts as a receptor for retinol-binding protein responsible for the cellular uptake of vitamin A, which is critical to the normal development of the eyes.(42) Indeed mutations in STRA6 impairing this function lead to severe developmental abnormalities in the eyes such microphthalmia, anophthalmia and coloboma.(42, 43) SIX3, PRSS56 and PAX6 are also involved in the eye development. PAX6 has been labelled as the master control gene for the morphogenesis of the eye, and is regulated by the transcriptional regulator SIX3.(44) BCAS3 and RSPO3 are involved in angiogenesis, vascular support and cell migration. $(45,46) B M P 4$ antagonises transforming growth factor-beta 2 (TGF- $\beta 2$ ) signalling, a cytoke involved in the synthesis and deposition of extracellular matrix in the optic nerve head.(47) This pathway is implicated in the pathological remodelling of the optic nerve head 
in glaucoma,(47) and deficiency of BMP4 results in an abnormal optic nerve with loose connective tissue.(48) All together, these gene findings help us have a better understanding of the development of the eye and related traits.

Optic disc size is highly correlated with the vertical cup-to-disc ratio (VCDR)(11), one of the main glaucoma endophenotypes, and is important for the interpretation of a glaucomatous optic disc.(5, 8) Clinically, adjusting VCDR to DD improves its utility as larger discs are more likely to have physiologically larger cups.(9) In clinical genetics, genes are more likely to be involved in the pathogenesis of glaucoma if associated with larger VCDR but not disc size, or VCDR adjusted to disc size. For instance, variation in the PDZD2 gene is associated with optic cup area and VCDR,(11) and our study identifies the same variation to be strongly associated with disc size. This would suggest that the observed association with VCDR is likely due to the disc size rather than a pathological enlargement of the optic cup. Similarly, the previously reported association between $F 5$ and VCDR is likely related to disc size due to its larger association with disc size in our study and previously.(16) When the disc size is adjusted for, Springelkamp et al. have reported the estimated effect size of the $F 5$ variant on VCDR is negligible.(11) Several of the identified disc size genes are correlated with intraocular pressure. For instance, genes TMEM119, CENPW, LTBP1, TEX41, and PKIA are reported to be associated with intraocular pressure, $(38,49-51)$ which could represent pleiotropic effects of these genes. Correlating disc size loci with the genes for glaucoma and its endophenotypes would help to identify the role of these genes in glaucoma pathogenesis.

Understanding the genetics of optic disc size will also contribute towards the understanding of the etiology of myopia. $(13,52)$ The clinical morphology of the optic disc in myopic eye is distinct and the disc size correlates with the magnitude of the refractive error especially in high myopes.(53) From the genetic correlation results using LD Hub, (27) we found disc size is negatively correlated with myopia $\left(\mathrm{rg}=-0.24, \mathrm{P}=5.94 \times 10^{-8}\right)$, and positively correlated with hypermetropia $\left(\mathrm{rg}=0.24, \mathrm{P}=9.18 \times 10^{-5}\right)$. Several novel disc size associated SNPs discovered in this study have been previously reported to be associated with myopia. Of these, variant rs74764079 located in the exonic region of bone morphogenetic protein 3 (BMP3) had a large CADD score (24.5). The Serine Protease 56 (PRSS56) and Bone Morphogenetic Protein 4 (BMP4) genes are involved in ocular growth as variations in these genes are associated with microphthalmia (abnormally small eyes).(5456) Variations in PRSS56 and BMP4 are reported to be associated with myopia in previous GWAS. $(57,58)$ 
Our results support the involvement of these genes in optic disc morphology. The tetraspanin 10 (TSPAN10) gene is involved in cellular protein trafficking and regulation and organ development.(59) Variations in TSPAN10 and NPLOC4 are associated with myopia and macular thickness (derived from ocular coherence tomography $[\mathrm{OCT}]$ scans).(60, 61) The association with optic disc size further supports their involvement in eye development.

There are several limitations for this study. Firstly, we only evaluated individuals of European ancestry in our disc size GWAS, hence the generalizability of the genetic findings to other populations remains unclear. However, the concordance in lead SNP effect sizes was also very high between UKBB disc size GWAS and IGGC disc size GWAS in Asian population (Pearson's correlation coefficient 0.72, Supplementary Figure S8). Another limitation is that in UKBB we measured the vertical disc diameter rather than disc area (measured in IGGC dataset). However, in our UKBB GWAS, we used the rank-based inverse-normal transformed disc diameter, which we subsequently rescaled to disc area (Supplementary Methods).(62, 63) In our sensitivity analysis, we used multiple trait analysis of GWAS (MTAG) to joint analyse UKBB and IGGC disc size summary statistics; the results are essentially identical to traditional inverse-variance meta-analysis (Supplementary Table S3, Figure S9, Supplementary Methods). Nonetheless, the identified novel loci and genes from meta-analysis and gene-based analysis still need further studies to replicate these findings. A third limitation is that disc size was not available for both the left and right eyes in all cohorts. For instance due to the lengthy manual process of grading 67,040 UKBB fundus photos, we graded the DD on the left eye where the image quality was good, otherwise the right eye was used; however, the DD between both eyes are expected to be very similar. In the EPIC-Norfolk sample set $(\mathrm{N}=6,005)$, the measurement of the vertical disc diameter was $2.34 \pm 0.26 \mathrm{~mm}$ in the right eyes, compared $2.33 \pm 0.26 \mathrm{~mm}$ in the left eyes, which is also consistent with previous studies.(64) A fourth limitation is that although differences in ocular magnification and tilted appearance of some optic discs could affect disc size measurements, in practice the effects of these are expected to be small (65). In our sensitivity analysis, we adjusted spherical equivalent refractive error in UKBB disc size GWAS; the results were essentially unchanged.

In conclusion, we conducted a meta-analysis GWAS of 95,549 individuals for disc size and identified 101 genomic loci across 159 genes. This study enhanced our understanding of the genetics of disc size and would shed light on the genetic findings for other eye traits such as VCDR, intraocular pressure, POAG, and myopia. 


\section{Materials and Methods}

Study procedures were performed in accordance with the World Medical Association Declaration of Helsinki ethical principles for medical research.

\section{UK Biobank disc diameter phenotype data}

UK Biobank is a large-scale cohort study that included over half a million participants aged between 40-69 years in 2006-2010 from across the United Kingdom. In the UKBB, 87,685 left fundus retinal eye images were available (two assessment visits), covering 84,871 participants (UKBB Field: 21015). The longest vertical DD was measured at the inner edges of the scleral ring from non-stereo fundus images obtained using a Topcon 3D OCT-1000 MKII (Topcon Corporation). The images had a $45^{\circ}$ field of view and were cropped and enlarged to facilitate grading using a custom Java program.

Two thousand images were randomly selected for quality control, and the Pearson's correlation coefficient of the DD measurements between the two examiners was 0.64 (95\% confidence interval $[\mathrm{CI}]: 0.61-0.67$; supplementary Figure S10). The second visit DD measurements were used if available, otherwise, we used the first visit measurements $(\mathrm{N}=52,199$, proportion $76 \%)$. If the left eye images were ungradable, we used the right eye images instead ( $\mathrm{N}=6,181$, proportion 9\%, UKBB Field 21016). In DD GWAS, we excluded non-white British ancestry participants based on principal components (PCs). Finally, 67,040 participants were included in our analysis. For statistical analysis, we applied a rank-based inverse-normal transformation to DD, effectively rendering the results in terms of disc area (see statistical methods, below).

\section{UK Biobank genotype data}

Detailed information of the genotype data and quality control procedures for UKBB was reported by Bycroft and colleagues.(66) Briefly, approximately 488,000 participants were genotyped for 805,426 markers on Axiom arrays (Affymetrix Santa Clara, USA). After standard quality control procedures, $~ 96 \mathrm{M}$ genotypes were imputed using Haplotype Reference Consortium (HRC) and UK10K haplotype resources.(66-68) In the association analysis, we removed single nucleotide polymorphisms (SNPs) with minor allele frequency (MAF) less than 0.01 or imputation quality score less than 0.3 . Finally, 8,928,767 SNPs were kept for association analysis. 


\section{IGGC disc size summary statistics}

The phenotype and genotype data of disc size for IGGC have been previously described elsewhere.(11) We downloaded the publicly available disc area GWAS summary statistics for 22,504 individuals of European ancestry from IGGC. In IGGC, the genetic data was imputed to 1000 Genomes reference panel, and the disc area GWAS was adjusted for age, sex and the first five principal components.(11)

\section{EPIC-Norfolk Eye Study}

The European Prospective Investigation into Cancer (EPIC) study is a pan-European prospective cohort study designed to investigate the aetiology of major chronic diseases.(69) EPIC-Norfolk, one of the UK arms of EPIC, recruited and examined 25,639 participants between 1993 and 1997 for the baseline examination.(70) Recruitment was via general practices in the city of Norwich and the surrounding small towns and rural areas, and methods have been described in detail previously. Ophthalmic assessment formed part of the third health examination and this has been termed the EPIC-Norfolk Eye Study.(71)

In total, 8,623 participants were seen for the Eye Study between 2004 and 2011. Digital photographs of the optic disc and macula were taken using a TRC-NW6S non- mydriatic retinal camera and IMAGEnet Telemedicine System (Topcon Corporation, Tokyo, Japan) with a 10-megapixel Nikon D80 camera (Nikon Corporation, Tokyo, Japan). Pupils were not dilated. Images were graded at the Moorfields Reading Centre. Measurement of the vertical diameter of the optic disc was made using adobe photoshop C55 software.

99.7\% of EPIC-Norfolk are of European descent and we excluded participants of non-white European ancestries. The EPIC-Norfolk Eye Study was carried out following the principles of the Declaration of Helsinki and the Research Governance Framework for Health and Social Care. The study was approved by the Norfolk Local Research Ethics Committee (05/Q0101/191) and East Norfolk \& Waveney NHS Research Governance Committee (2005EC07L). All participants gave written, informed consent.

Initial genotyping on a small subset of EPIC-Norfolk was undertaken using the Affymetrix GeneChip Human Mapping 500K Array Set and 1,096 of these participants contributed to the IGGC meta-analysis.(11) Subsequently, the rest of the EPIC-Norfolk cohort were genotyped using the Affymetrix UK Biobank Axiom Array (the same array as used in UK Biobank); it is 6,005 of these participants (which includes no overlap with the 1,096 participants contributing to the IGGC meta-analysis(11)) that contributed to the EPIC-Norfolk 
disc size GWAS in the current study. SNP exclusion criteria included: call rate $<95 \%$, abnormal cluster pattern on visual inspection, plate batch effect evident by significant variation in minor allele frequency, and/or HardyWeinberg equilibrium $P<10^{-7}$. Sample exclusion criteria included: DishQC $<0.82$ (poor fluorescence signal contrast), sex discordance, sample call rate $<97 \%$, heterozygosity outliers (calculated separately for SNPs with minor allele frequency $>1 \%$ and $=<1 \%$ ), rare allele count outlier, and implausible identity-by-descent values. We removed related individuals with pairwise relatedness corresponding to third-degree relatives or closer across all genotyped participants. Following these exclusions, there were no ethnic outliers. Data were prephased using SHAPEIT version 2 and imputed to the Phase 3 build of the 1000 Genomes project (October 2014) using IMPUTE (version 2.3.2).

We examined the relationship between allele dosage and mean of right and left vertical disc diameter using linear regression adjusted for age, sex and the first five principal components. Analyses were carried out using SNPTEST software (version 2.5.1).(72)

\section{Ocular gene expression analysis}

Gene expression data was available from RNA extraction of 21 healthy donor eyes from 21 individuals. We analysed 63 tissues of cornea (epithelium, stroma and endothelium), trabecular meshwork, ciliary body, iris, retina, optic nerve and optic nerve head. RNA quality was assessed using Agilent Bioanalyzer 2100 RNA 6000 Nano Assay and samples were included for sequencing only if the RIN scores were greater than or equal to 3.8 and both $28 \mathrm{~S}$ and $18 \mathrm{~S}$ ribosomal RNA intensity peaks were prominent. RNA sequencing was done using Illumina NextSeq® 500 (San Diego, USA), followed by quality check (FASTQC v0.11.3). Trimgalore (v0.4.0) was used to trim low quality bases (Phred score < 28) and reads shorter than 20 bases after trimming were discarded. All reads which passed every quality control step were then aligned to the human genome (GRCh38 assembly) with $\leq 2$ mismatches per read. Downstream analysis was done with edgeR (version 3.22.5).(73) We selected genes expressed 10 times (1.5 counts per million) in at least 5 tissue samples and normalised the libraries using trimmed mean of M-values (TMM).(74) Estimating dispersions was done via Cox-Reid profileadjusted likelihood method,(75) and differential expression was compared between optic nerve head and all other tissues via negative binomial generalised linear model.(76) Genes were filtered to those nearest to the identified SNPs, and the differential expression P-values were adjusted using Bonferroni correction. 


\section{Genome-wide association analysis and meta-analysis}

For DD GWAS in UKBB, we first applied a rank-based inverse-normal transformation.(63) Since disc diameter and disc area are monotonically related, applying such a transformation makes the phenotype correlation between diameter and area effectively approach one, although to render them back to the same output scale, one should multiply by the standard deviation of the trait, which is approximately $0.4 \mathrm{~mm}^{2}$ for disc area.(11) To ensure consistency with previously reported results, all our analyses are presented on the disc area scale.(11) For association analysis in UKBB, we used a linear mixed model in BOLT-LMM software (version 2.3).(77) Analysis was performed under an additive genetic model, adjusted for the effect of sex, age, the first ten principal components, two indicator variables for examiners who performed the measurements, and fundus retinal image assessment visits. (84) A stepwise model selection procedure in the GCTA-COJO software (version 1.91.7beta) was used to identify independent lead genome-wide significant SNPs.(78) We reported independent SNPs with both unconditional $\mathrm{P}$ values and joint $\mathrm{P}$ values less than $5 \times 10^{-8}$. For genomic regions with multiple independent SNPs, we defined a 'locus' as a region at least 400 kilobases from the adjacent locus. Bivariate linkage disequilibrium (LD) score regression was used to estimate the genetic correlation between pairs of traits.(79) To replicate the lead SNPs from UKBB, we conducted a sample size based meta-analysis in METAL (2011-03-25 release) for IGGC and EPIC-Norfolk datasets.(80) For the UKBB and IGGC meta-analysis, we performed the inverse-variance weighted fixed-effect meta-analysis in METAL.(80) In our sensitivity analysis, rather than performing meta-analysis using the effect size estimates and standard errors, we also conducted the multiple trait analysis of GWAS (MTAG, software version 1.08) approach, a framework to generalize the standard inverse-variance meta-analysis method, with the approach able to joint analyse the same trait with different measures or even different traits with a high genetic correlation.(81) The general analyses were performed with R software (version 3.4.1).(82) Additional details are provided in supplementary Methods.

\section{Gene-based and pathway tests}

We used MAGMA (v1.07) for gene-based and pathway analysis as implemented in FUMA (version 1.3.4).(21,

83) In gene-based tests, GWAS summary statistics of SNPs were mapped to 18,619 genes, and the association $\mathrm{P}$ values for a set of SNPs were calculated. The default parameters in FUMA were used. Bonferroni method 
was used for multiple testing correction $(\mathrm{P}<0.05 / 18,619)$. In pathway tests, 10,678 predefined gene sets (MsigDB v6.2, curated gene sets: 4,761, GO terms: 5,917) were tested for enrichment.

\section{Functional annotation}

The functional annotation of SNPs was implemented in FUMA (version 1.3.4).(21) Firstly, the functional annotation of SNPs on the genes were obtained from ANNOVAR.(84) The detailed annotations included downstream, exonic, intergenic, and upstream. We then checked the chromatin states of the SNPs. The 15core chromatin states were predicted by ChromHMM software based on 5 chromatin marks for 127 epigenomes.(20) We also looked up the Regulome database score and CADD score.(22, 85) Briefly, the Regulome database score represents the evidence of regulatory function of SNPs based on eQTLs and chromatin marks. The highest score 1a means that those SNPs may affect regulatory elements while score 7 means not regulatory. CADD score is an integrative metric to measure variant deleteriousness of SNPs predicted by 63 functional annotations. The higher the score, the more likely that a SNP is deleteriousness (suggested threshold 12.37),(22) which could be used to prioritize causal variations.

\section{eQTL lookup and SMR method}

The lead DD SNPs were looked up in the Eye Genotype Expression (EyeGEx) database of retinal tissue to identify retina specific expression quantitative trait loci (eQTL).(32) We also applied SMR (summary databased Mendelian randomization) and HEIDI (heterogeneity in dependent instruments) tests based on DD metaanalysis summary statistics and the EyeGEx eQTL data.(33) The SMR approach uses both of GWAS summary statistics and eQTL data to test if the effect of a SNP on the phenotype is mediated by gene expression, which could be used to prioritize GWAS hits for further functional studies. The HEIDI method can test the null hypothesis that there is a single causal variant affecting both gene expression levels and phenotype risk. 


\section{Acknowledgements}

This work was conducted using the UK Biobank Resource (application number 25331) and publicly available data from the International Glaucoma Genetics Consortium. SM, JEC and AWH are supported by NHMRC Fellowships. SM was supported by an Australian Research Council Future Fellowship. XH is supported by the University of Queensland Research Training Scholarship and QIMR Berghofer PhD Top Up Scholarship. We thank Scott Wood, Xiaping Lin, John Pearson and Scott Gordon from QIMR Berghofer for support.

\section{Conflict of interest disclosures}

The authors declare no potential conflicts of interest. 


\section{References}

1. Quigley,H.A. (2011) Glaucoma. Lancet, 377, 1367-1377.

2. Cekić,S., Stanković-Babić,G., Visnjić,Z., Jovanović,I. and Risimić,D. (2010) Optic disc abnormalities diagnosis, evolution and influence on visual acuity. Bosn. J. Basic Med. Sci., 10, 125-132.

3. Casson,R.J., Chidlow,G., Wood,J.P.M., Crowston,J.G. and Goldberg,I. (2012) Definition of glaucoma: clinical and experimental concepts. Clin. Experiment. Ophthalmol., 40, 341-349.

4. Foster,P.J., Buhrmann,R., Quigley,H.A. and Johnson,G.J. (2002) The definition and classification of glaucoma in prevalence surveys. Br. J. Ophthalmol., 86, 238-242.

5. Hoffmann,E.M., Zangwill,L.M., Crowston,J.G. and Weinreb,R.N. (2007) Optic disk size and glaucoma. Surv. Ophthalmol., 52, 32-49.

6. Li,A., Li,L., Li,M. and Shi,X. (2015) A new characterization for nonarteritic anterior ischemic optic neuropathy. Int. J. Clin. Exp. Med., 8, 18681-18688.

7. Chang,M.Y. and Pineles,S.L. (2016) Optic disk drusen in children. Surv. Ophthalmol., 61, 745-758.

8. Crowston,J.G., Hopley,C.R., Healey,P.R., Lee,A., Mitchell,P. and Blue Mountains Eye Study (2004) The effect of optic disc diameter on vertical cup to disc ratio percentiles in a population based cohort: the Blue Mountains Eye Study. Br. J. Ophthalmol., 88, 766-770.

9. Jonas,J.B., Bergua,A., Schmitz-Valckenberg,P., Papastathopoulos,K.I. and Budde,W.M. (2000) Ranking of optic disc variables for detection of glaucomatous optic nerve damage. Invest. Ophthalmol. Vis. Sci., 41, 1764-1773.

10. Klein,B.E.K., Klein,R. and Lee,K.E. (2004) Heritability of risk factors for primary open-angle glaucoma: the Beaver Dam Eye Study. Invest. Ophthalmol. Vis. Sci., 45, 59-62.

11. Springelkamp,H., Iglesias,A.I., Mishra,A., Hohn,R., Wojciechowski,R., Khawaja,A.P., Nag,A., Wang,Y.X., Wang,J.J., Cuellar-Partida,G., et al. (2017) New insights into the genetics of primary openangle glaucoma based on meta-analyses of intraocular pressure and optic disc characteristics. Hum. Mol. Genet., 26, 438-453.

12. Macgregor,S., Hewitt,A.W., Hysi,P.G., Ruddle,J.B., Medland,S.E., Henders,A.K., Gordon,S.D., Andrew,T., McEvoy,B., Sanfilippo,P.G., et al. (2010) Genome-wide association identifies ATOH7 as a major gene determining human optic disc size. Hum. Mol. Genet., 19, 2716-2724.

13. Ramdas,W.D., van Koolwijk,L.M.E., Ikram,M.K., Jansonius,N.M., de Jong,P.T.V.M., Bergen,A.A.B., Isaacs,A., Amin,N., Aulchenko,Y.S., Wolfs,R.C.W., et al. (2010) A genome-wide association study of optic disc parameters. PLoS Genet., 6, e1000978.

14. Khor,C.C., Ramdas,W.D., Vithana,E.N., Cornes,B.K., Sim,X., Tay,W.-T., Saw,S.-M., Zheng,Y., Lavanya,R., Wu,R., et al. (2011) Genome-wide association studies in Asians confirm the involvement of ATOH7 and TGFBR3, and further identify CARD10 as a novel locus influencing optic disc area. Hum. Mol. Genet., 20, 1864-1872.

15. Gasten,A.C., Ramdas,W.D., Broer,L., van Koolwijk,L.M., Ikram,M.K., de Jong,P.T., Aulchenko,Y.S., Wolfs,R.C., Hofman,A., Rivadeneira,F., et al. (2012) A genetic epidemiologic study of candidate genes involved in the optic nerve head morphology. Invest. Ophthalmol. Vis. Sci., 53, 1485-1491.

16. Springelkamp,H., Mishra,A., Hysi,P.G., Gharahkhani,P., Höhn,R., Khor,C.-C., Cooke Bailey,J.N., Luo,X., Ramdas,W.D., Vithana,E., et al. (2015) Meta-analysis of Genome-Wide Association Studies Identifies Novel Loci Associated With Optic Disc Morphology. Genet. Epidemiol., 39, 207-216.

17. Nagai,T., Aruga,J., Takada,S., Günther,T., Spörle,R., Schughart,K. and Mikoshiba,K. (1997) The expression of the mouse Zic1, Zic2, and Zic3 gene suggests an essential role for Zic genes in body pattern formation. Dev. Biol., 182, 299-313. 
18. Cavodeassi,F., Ivanovitch,K. and Wilson,S.W. (2013) Eph/Ephrin signalling maintains eye field segregation from adjacent neural plate territories during forebrain morphogenesis. Development, 140, 4193-4202.

19. Jonas,J.B., Schmidt,A.M., Müller-Bergh,J.A., Schlötzer-Schrehardt,U.M. and Naumann,G.O. (1992) Human optic nerve fiber count and optic disc size. Invest. Ophthalmol. Vis. Sci., 33, 2012-2018.

20. Ernst,J. and Kellis,M. (2012) ChromHMM: automating chromatin-state discovery and characterization. Nat. Methods, 9, 215-216.

21. Watanabe,K., Taskesen,E., van Bochoven,A. and Posthuma,D. (2017) Functional mapping and annotation of genetic associations with FUMA. Nat. Commun., 8, 1826.

22. Kircher,M., Witten,D.M., Jain,P., O'Roak,B.J., Cooper,G.M. and Shendure,J. (2014) A general framework for estimating the relative pathogenicity of human genetic variants. Nat. Genet., 46, 310315.

23. Rentzsch,P., Witten,D., Cooper,G.M., Shendure,J. and Kircher,M. (2019) CADD: predicting the deleteriousness of variants throughout the human genome. Nucleic Acids Res., 47, D886-D894.

24. Cha,S., Lim,J.E., Park,A.Y., Do,J.-H., Lee,S.W., Shin,C., Cho,N.H., Kang,J.-O., Nam,J.M., Kim,J.-S., et al. (2018) Identification of five novel genetic loci related to facial morphology by genome-wide association studies. BMC Genomics, 19, 481.

25. Ramjaun,A.R., Tomlinson,S., Eddaoudi,A. and Downward,J. (2007) Upregulation of two BH3-only proteins, Bmf and Bim, during TGF beta-induced apoptosis. Oncogene, 26, 970-981.

26. Saika,S. (2006) TGFbeta pathobiology in the eye. Lab. Invest., 86, 106-115.

27. Zheng,J., Erzurumluoglu,A.M., Elsworth,B.L., Kemp,J.P., Howe,L., Haycock,P.C., Hemani,G., Tansey,K., Laurin,C., Early Genetics and Lifecourse Epidemiology (EAGLE) Eczema Consortium, et al. (2017) LD Hub: a centralized database and web interface to perform LD score regression that maximizes the potential of summary level GWAS data for SNP heritability and genetic correlation analysis. Bioinformatics, 33, 272-279.

28. Morales,J., Welter,D., Bowler,E.H., Cerezo,M., Harris,L.W., McMahon,A.C., Hall,P., Junkins,H.A., Milano,A., Hastings,E., et al. (2018) A standardized framework for representation of ancestry data in genomics studies, with application to the NHGRI-EBI GWAS Catalog. Genome Biol., 19, 21.

29. Hoshino,A., Ratnapriya,R., Brooks,M.J., Chaitankar,V., Wilken,M.S., Zhang,C., Starostik,M.R., Gieser,L., La Torre,A., Nishio,M., et al. (2017) Molecular Anatomy of the Developing Human Retina. Dev. Cell, 43, 763-779.e4.

30. Haeseleer,F. and Palczewski,K. (2000) [24] Short-chain dehydrogenases/reductases in retina. In Methods in Enzymology. Academic Press, Vol. 316, pp. 372-383.

31. Gharahkhani,P., Burdon,K.P., Cooke Bailey,J.N., Hewitt,A.W., Law,M.H., Pasquale,L.R., Kang,J.H., Haines,J.L., Souzeau,E., Zhou,T., et al. (2018) Analysis combining correlated glaucoma traits identifies five new risk loci for open-angle glaucoma. Sci. Rep., $8,3124$.

32. Ratnapriya,R., Sosina,O.A., Starostik,M.R., Kwicklis,M., Kapphahn,R.J., Fritsche,L.G., Walton,A., Arvanitis,M., Gieser,L., Pietraszkiewicz,A., et al. (2019) Retinal transcriptome and eQTL analyses identify genes associated with age-related macular degeneration. Nat. Genet., 51, 606-610.

33. Zhu,Z., Zhang,F., Hu,H., Bakshi,A., Robinson,M.R., Powell,J.E., Montgomery,G.W., Goddard,M.E., Wray,N.R., Visscher,P.M., et al. (2016) Integration of summary data from GWAS and eQTL studies predicts complex trait gene targets. Nat. Genet., 48, 481-487.

34. Iliescu,A., Gravel,M., Horth,C., Apuzzo,S. and Gros,P. (2011) Transmembrane topology of mammalian planar cell polarity protein Vangl1. Biochemistry, 50, 2274-2282.

35. Belotti,E., Puvirajesinghe,T.M., Audebert,S., Baudelet,E., Camoin,L., Pierres,M., Lasvaux,L., 
Ferracci,G., Montcouquiol,M. and Borg,J.-P. (2012) Molecular characterisation of endogenous Vangl2/Vangl1 heteromeric protein complexes. PLoS One, 7, e46213.

36. Wolff,T. and Rubin,G.M. (1998) Strabismus, a novel gene that regulates tissue polarity and cell fate decisions in Drosophila. Development, 125, 1149-1159.

37. Prendergast,L., van Vuuren,C., Kaczmarczyk,A., Doering,V., Hellwig,D., Quinn,N., Hoischen,C., Diekmann,S. and Sullivan,K.F. (2011) Premitotic assembly of human CENPs -T and -W switches centromeric chromatin to a mitotic state. PLoS Biol., 9, e1001082.

38. Gao,X.R., Huang,H., Nannini,D.R., Fan,F. and Kim,H. (2018) Genome-Wide Association Analyses Identify New Loci Influencing Intraocular Pressure. Hum. Mol. Genet., 10.1093/hmg/ddy111.

39. N’Diaye,A., Chen,G.K., Palmer,C.D., Ge,B., Tayo,B., Mathias,R.A., Ding,J., Nalls,M.A., Adeyemo,A., Adoue, $V$., et al. (2011) Identification, replication, and fine-mapping of Loci associated with adult height in individuals of african ancestry. PLoS Genet., 7, e1002298.

40. Shungin,D., Winkler,T.W., Croteau-Chonka,D.C., Ferreira,T., Locke,A.E., Mägi,R., Strawbridge,R.J., Pers,T.H., Fischer,K., Justice,A.E., et al. (2015) New genetic loci link adipose and insulin biology to body fat distribution. Nature, 518, 187-196.

41. Elks,C.E., Perry,J.R.B., Sulem,P., Chasman,D.I., Franceschini,N., He,C., Lunetta,K.L., Visser,J.A., Byrne,E.M., Cousminer,D.L., et al. (2010) Thirty new loci for age at menarche identified by a metaanalysis of genome-wide association studies. Nat. Genet., 42, 1077-1085.

42. Casey,J., Kawaguchi,R., Morrissey,M., Sun,H., McGettigan,P., Nielsen,J.E., Conroy,J., Regan,R., Kenny,E., Cormican,P., et al. (2011) First implication of STRA6 mutations in isolated anophthalmia, microphthalmia, and coloboma: a new dimension to the STRA6 phenotype. Hum. Mutat., 32, 14171426.

43. Pasutto,F., Sticht,H., Hammersen,G., Gillessen-Kaesbach,G., Fitzpatrick,D.R., Nürnberg,G., Brasch,F., Schirmer-Zimmermann,H., Tolmie,J.L., Chitayat,D., et al. (2007) Mutations in STRA6 cause a broad spectrum of malformations including anophthalmia, congenital heart defects, diaphragmatic hernia, alveolar capillary dysplasia, lung hypoplasia, and mental retardation. Am. J. Hum. Genet., 80, 550-560.

44. Azuma,N., Yamaguchi,Y., Handa,H., Tadokoro,K., Asaka,A., Kawase,E. and Yamada,M. (2003) Mutations of the PAX6 gene detected in patients with a variety of optic-nerve malformations. Am. J. Hum. Genet., 72, 1565-1570.

45. Jain,M., Bhat,G.P., Vijayraghavan,K. and Inamdar,M.S. (2012) Rudhira/BCAS3 is a cytoskeletal protein that controls $\mathrm{Cdc} 42$ activation and directional cell migration during angiogenesis. Exp. Cell Res., 318, $753-767$.

46. Kazanskaya,O., Ohkawara,B., Heroult,M., Wu,W., Maltry,N., Augustin,H.G. and Niehrs,C. (2008) The Wnt signaling regulator R-spondin 3 promotes angioblast and vascular development. Development, 135, 3655-3664.

47. Zode,G.S., Clark,A.F. and Wordinger,R.J. (2009) Bone morphogenetic protein 4 inhibits TGF-beta2 stimulation of extracellular matrix proteins in optic nerve head cells: role of gremlin in ECM modulation. Glia, 57, 755-766.

48. Chang,B., Smith,R.S., Peters,M., Savinova,O.V., Hawes,N.L., Zabaleta,A., Nusinowitz,S., Martin,J.E., Davisson,M.L., Cepko,C.L., et al. (2001) Haploinsufficient Bmp4 ocular phenotypes include anterior segment dysgenesis with elevated intraocular pressure. BMC Genet., 2, 18.

49. MacGregor,S., Ong,J.-S., An,J., Han,X., Zhou,T., Siggs,O.M., Law,M.H., Souzeau,E., Sharma,S., Lynn,D.J., et al. (2018) Genome-wide association study of intraocular pressure uncovers new pathways to glaucoma. Nat. Genet., 50, 1067-1071.

50. Khawaja,A.P., Cooke Bailey,J.N., Wareham,N.J., Scott,R.A., Simcoe,M., Igo,R.P.,Jr, Song,Y.E., Wojciechowski,R., Cheng,C.-Y., Khaw,P.T., et al. (2018) Genome-wide analyses identify 68 new loci 
associated with intraocular pressure and improve risk prediction for primary open-angle glaucoma. Nat. Genet., 50, 778-782.

51. Choquet,H., Thai,K.K., Yin,J., Hoffmann,T.J., Kvale,M.N., Banda,Y., Schaefer,C., Risch,N., Nair,K.S., Melles,R., et al. (2017) A large multi-ethnic genome-wide association study identifies novel genetic loci for intraocular pressure. Nat. Commun., 8, 2108.

52. Leung,C.K.-S., Cheng,A.C.K., Chong,K.K.L., Leung,K.S., Mohamed,S., Lau,C.S.L., Cheung,C.Y.L., Chu,G.C.-H., Lai,R.Y.K., Pang,C.C.P., et al. (2007) Optic disc measurements in myopia with optical coherence tomography and confocal scanning laser ophthalmoscopy. Invest. Ophthalmol. Vis. Sci., 48, $3178-3183$.

53. Jonas,J.B., Gusek,G.C. and Naumann,G.O. (1988) Optic disk morphometry in high myopia. Graefes Arch. Clin. Exp. Ophthalmol., 226, 587-590.

54. Orr,A., Dubé,M.-P., Zenteno,J.C., Jiang,H., Asselin,G., Evans,S.C., Caqueret,A., Lakosha,H., Letourneau,L., Marcadier,J., et al. (2011) Mutations in a novel serine protease PRSS56 in families with nanophthalmos. Mol. Vis., 17, 1850-1861.

55. Paylakhi,S., Labelle-Dumais,C., Tolman,N.G., Sellarole,M.A., Seymens,Y., Saunders,J., Lakosha,H., deVries,W.N., Orr,A.C., Topilko,P., et al. (2018) Müller glia-derived PRSS56 is required to sustain ocular axial growth and prevent refractive error. PLoS Genet., 14, e1007244.

56. Reis,L.M., Tyler,R.C., Schilter,K.F., Abdul-Rahman,O., Innis,J.W., Kozel,B.A., Schneider,A.S., Bardakjian,T.M., Lose,E.J., Martin,D.M., et al. (2011) BMP4 loss-of-function mutations in developmental eye disorders including SHORT syndrome. Hum. Genet., 130, 495-504.

57. Verhoeven,V.J.M., Hysi,P.G., Wojciechowski,R., Fan,Q., Guggenheim,J.A., Höhn,R., MacGregor,S., Hewitt,A.W., Nag,A., Cheng,C.-Y., et al. (2013) Genome-wide meta-analyses of multiancestry cohorts identify multiple new susceptibility loci for refractive error and myopia. Nat. Genet., 45, 314-318.

58. Kiefer,A.K., Tung,J.Y., Do,C.B., Hinds,D.A., Mountain,J.L., Francke,U. and Eriksson,N. (2013) Genome-wide analysis points to roles for extracellular matrix remodeling, the visual cycle, and neuronal development in myopia. PLoS Genet., 9, e1003299.

59. Charrin,S., Jouannet,S., Boucheix,C. and Rubinstein,E. (2014) Tetraspanins at a glance. J. Cell Sci., 127, 3641-3648.

60. Pickrell,J.K., Berisa,T., Liu,J.Z., Ségurel,L., Tung,J.Y. and Hinds,D.A. (2016) Detection and interpretation of shared genetic influences on 42 human traits. Nat. Genet., 48, 709-717.

61. Gao,X.R., Huang,H. and Kim,H. (2018) Genome-wide association analyses identify 139 loci associated with macular thickness in the UK Biobank cohort. Hum. Mol. Genet., 10.1093/hmg/ddy422.

62. Bartling,H., Wanger,P. and Martin,L. (2008) Measurement of optic disc parameters on digital fundus photographs: algorithm development and evaluation. Acta Ophthalmol., 86, 837-841.

63. Aulchenko,Y.S., Ripke,S., Isaacs,A. and van Duijn,C.M. (2007) GenABEL: an R library for genomewide association analysis. Bioinformatics, 23, 1294-1296.

64. Quigley,H.A., Brown,A.E., Morrison,J.D. and Drance,S.M. (1990) The size and shape of the optic disc in normal human eyes. Arch. Ophthalmol., 108, 51-57.

65. Garway-Heath,D.F., Rudnicka,A.R., Lowe,T., Foster,P.J., Fitzke,F.W. and Hitchings,R.A. (1998) Measurement of optic disc size: equivalence of methods to correct for ocular magnification. Br. J. Ophthalmol., 82, 643-649.

66. Bycroft,C., Freeman,C., Petkova,D., Band,G., Elliott,L.T., Sharp,K., Motyer,A., Vukcevic,D., Delaneau,O., O'Connell,J., et al. (2018) The UK Biobank resource with deep phenotyping and genomic data. Nature, 562, 203-209.

67. McCarthy,S., Das,S., Kretzschmar,W., Delaneau,O., Wood,A.R., Teumer,A., Kang,H.M., 
Fuchsberger,C., Danecek,P., Sharp,K., et al. (2016) A reference panel of 64,976 haplotypes for genotype imputation. Nat. Genet., 48, 1279-1283.

68. UK10K Consortium, Walter,K., Min,J.L., Huang,J., Crooks,L., Memari,Y., McCarthy,S., Perry,J.R.B., $\mathrm{Xu}, \mathrm{C}$., Futema,M., et al. (2015) The UK10K project identifies rare variants in health and disease. Nature, 526, 82-90.

69. Riboli,E. and Kaaks,R. (1997) The EPIC Project: rationale and study design. European Prospective Investigation into Cancer and Nutrition. Int. J. Epidemiol., 26 Suppl 1, S6-14.

70. Day,N., Oakes,S., Luben,R., Khaw,K.T., Bingham,S., Welch,A. and Wareham,N. (1999) EPIC-Norfolk: study design and characteristics of the cohort. European Prospective Investigation of Cancer. $\mathrm{Br} . J$. Cancer, 80 Suppl 1, 95-103.

71. Khawaja,A.P., Chan,M.P.Y., Hayat,S., Broadway,D.C., Luben,R., Garway-Heath,D.F., Sherwin,J.C., Yip,J.L.Y., Dalzell,N., Wareham,N.J., et al. (2013) The EPIC-Norfolk Eye Study: rationale, methods and a cross-sectional analysis of visual impairment in a population-based cohort. BMJ Open, $\mathbf{3}$, e002684.

72. Marchini,J., Howie,B., Myers,S., McVean,G. and Donnelly,P. (2007) A new multipoint method for genome-wide association studies by imputation of genotypes. Nat. Genet., 39, 906-913.

73. Robinson,M.D., McCarthy,D.J. and Smyth,G.K. (2010) edgeR: a Bioconductor package for differential expression analysis of digital gene expression data. Bioinformatics, 26, 139-140.

74. Robinson,M.D. and Oshlack,A. (2010) A scaling normalization method for differential expression analysis of RNA-seq data. Genome Biol., 11, R25.

75. Cox,D.R. and Reid,N. (1987) Parameter Orthogonality and Approximate Conditional Inference. J. R. Stat. Soc. Series B Stat. Methodol., 49, 1-18.

76. McCarthy,D.J., Chen,Y. and Smyth,G.K. (2012) Differential expression analysis of multifactor RNA-Seq experiments with respect to biological variation. Nucleic Acids Res., 40, 4288-4297.

77. Loh,P.-R., Tucker,G., Bulik-Sullivan,B.K., Vilhjálmsson,B.J., Finucane,H.K., Salem,R.M., Chasman,D.I., Ridker,P.M., Neale,B.M., Berger,B., et al. (2015) Efficient Bayesian mixed-model analysis increases association power in large cohorts. Nat. Genet., 47, 284-290.

78. Yang,J., Ferreira,T., Morris,A.P., Medland,S.E., Genetic Investigation of ANthropometric Traits (GIANT) Consortium, DIAbetes Genetics Replication And Meta-analysis (DIAGRAM) Consortium, Madden,P.A.F., Heath,A.C., Martin,N.G., Montgomery,G.W., et al. (2012) Conditional and joint multiple-SNP analysis of GWAS summary statistics identifies additional variants influencing complex traits. Nat. Genet., 44, 369-75, S1-3.

79. Bulik-Sullivan,B., Finucane,H.K., Anttila,V., Gusev,A., Day,F.R., Loh,P.-R., ReproGen Consortium, Psychiatric Genomics Consortium, Genetic Consortium for Anorexia Nervosa of the Wellcome Trust Case Control Consortium 3, Duncan,L., et al. (2015) An atlas of genetic correlations across human diseases and traits. Nat. Genet., 47, 1236-1241.

80. Willer,C.J., Li,Y. and Abecasis,G.R. (2010) METAL: fast and efficient meta-analysis of genomewide association scans. Bioinformatics, 26, 2190-2191.

81. Turley,P., Walters,R.K., Maghzian,O., Okbay,A., Lee,J.J., Fontana,M.A., Nguyen-Viet,T.A., Wedow,R., Zacher,M., Furlotte,N.A., et al. (2018) Multi-trait analysis of genome-wide association summary statistics using MTAG. Nat. Genet., 50, 229-237.

82. R Core Team (2017) R: A Language and Environment for Statistical Computing.

83. de Leeuw,C.A., Mooij,J.M., Heskes,T. and Posthuma,D. (2015) MAGMA: generalized gene-set analysis of GWAS data. PLoS Comput. Biol., 11, e1004219.

84. Wang,K., Li,M. and Hakonarson,H. (2010) ANNOVAR: functional annotation of genetic variants from 
high-throughput sequencing data. Nucleic Acids Res., 38, e164.

85. Boyle,A.P., Hong,E.L., Hariharan,M., Cheng,Y., Schaub,M.A., Kasowski,M., Karczewski,K.J., Park,J., Hitz,B.C., Weng,S., et al. (2012) Annotation of functional variation in personal genomes using RegulomeDB. Genome Res., 22, 1790-1797. 


\section{Figure legends}

Figure 1. Manhattan plot of disc size genome-wide association studies.

A) UK Biobank dataset; B) UK Biobank and IGGC meta-analysis. Novel loci are highlighted in red dots, with the nearest gene names in black text. Known loci are highlighted in purple dots, with the nearest gene names in purple text. The red line is the genome-wide significance level $\left(\mathrm{P}=5 \times 10^{-8}\right)$.

Figure 2. Comparison of the effect sizes for 91 genome-wide significant independent SNPs identified from UK Biobank disc size GWAS versus those in independent cohort of IGGC disc size GWAS.

Pearson's correlation coefficient is $0.90\left(\mathrm{P}\right.$ value $\left.=2.85 \times 10^{-33}\right)$. The red line is the best fit line, with the $95 \%$ confidence interval region in grey. Novel disc size SNPs are highlighted in red and known SNPs in purple.

Figure 3. Functional annotation of genome-wide significant variants $\left(P<5 \times 10^{-8}\right)$.

A) functional categories; B) the minimum chromatin state across 127 tissues; C) the Regulome database score; D) the frequency of SNPs with high CADD score (more than the threshold 12.37). For A-C, the numbers and percentages in parentheses in the legends refer to the number of significant SNPs and the percentages in all identified significant variants. CADD, combined annotation dependent depletion. ncRNA, noncoding RNA; TF, transcription factor; TSS, transcription start site; UTR, untranslated region.

Table 1: List of 19 novel disc size loci replicated in IGGC and EPIC-Norfolk datasets after Bonferroni correction. 


\section{Abbreviations}

CADD, Combined Annotation Dependent Depletion

$\mathrm{DD}$, disc diameter

EPIC, European Prospective Investigation into Cancer

GWAS, genome-wide association study

HRC, Haplotype Reference Consortium

IGGC, International Glaucoma Genetic Consortium

MTAG, multiple trait analysis of GWAS

POAG, primary open angle glaucoma

SNP, single nucleotide polymorphism

UKBB, UK Biobank

VCDR, vertical cup-to-disc ratio 
Table 1: List of 19 novel disc size loci replicated in IGGC and EPIC-Norfolk datasets after Bonferroni correction.

\begin{tabular}{|c|c|c|c|c|c|c|c|c|c|c|}
\hline SNP & CHR & BP & Nearest Gene & EA & NEA & FREQ & $\begin{array}{c}\text { BETA } \\
\text { (UKBB) }\end{array}$ & $\begin{array}{c}\mathbf{P} \\
(\text { UKBB })\end{array}$ & $\begin{array}{c}\text { Z score } \\
\text { (META) }^{1}\end{array}$ & $\begin{array}{c}\mathbf{P} \\
\text { (META) }\end{array}$ \\
\hline rs12136690 & 1 & 116208944 & VANGL1 & $\mathrm{C}$ & $\mathrm{T}$ & 0.76 & -0.02 & $3.1 \mathrm{E}-24$ & 5.40 & 6.7E-08 \\
\hline rs56412756 & 1 & 201605477 & $N A V 1$ & $\mathrm{C}$ & $\mathrm{T}$ & 0.92 & 0.02 & $2.0 \mathrm{E}-08$ & -3.96 & 7.6E-05 \\
\hline rs9967780 & 2 & 56234942 & $M I R 216 B$ & G & $\mathrm{T}$ & 0.78 & -0.01 & 3.0E-09 & 4.73 & 2.3E-06 \\
\hline rs4832012 & 2 & 86000500 & АТОН8 & G & $\mathrm{C}$ & 0.49 & -0.01 & $1.4 \mathrm{E}-11$ & 3.69 & 2.3E-04 \\
\hline rs1365902 & 2 & 145470699 & TEX41 & $\mathrm{T}$ & $\mathrm{C}$ & 0.33 & -0.01 & 8.7E-12 & -4.91 & $9.1 \mathrm{E}-07$ \\
\hline rs3914468 & 2 & 170157400 & $L R P 2$ & A & G & 0.70 & -0.01 & $2.0 \mathrm{E}-10$ & -3.58 & 3.4E-04 \\
\hline rs77877421 & 3 & 71182447 & FOXP1 & A & $\mathrm{T}$ & 0.94 & -0.03 & 2.6E-09 & -3.47 & 5.1E-04 \\
\hline rs72759609 & 5 & 31952051 & PDZD2 & $\mathrm{T}$ & $\mathrm{C}$ & 0.90 & 0.03 & $3.1 \mathrm{E}-17$ & 4.02 & $5.9 \mathrm{E}-05$ \\
\hline rs58531939 & 5 & 87823968 & LINC00461 & $\mathrm{T}$ & $\mathrm{C}$ & 0.91 & -0.03 & $6.3 \mathrm{E}-16$ & -4.55 & 5.3E-06 \\
\hline rs2092524 & 6 & 39529692 & KIF6 & $\mathrm{G}$ & A & 0.66 & -0.01 & $8.5 \mathrm{E}-11$ & 4.18 & $3.0 \mathrm{E}-05$ \\
\hline rs12661045 & 6 & 122682795 & $H S F 2$ & $\mathrm{C}$ & $\mathrm{T}$ & 0.70 & 0.02 & $6.1 \mathrm{E}-14$ & -3.46 & $5.5 \mathrm{E}-04$ \\
\hline rs2152876 & 6 & 126761228 & CENPW & $\mathrm{G}$ & A & 0.54 & -0.02 & $1.5 \mathrm{E}-18$ & 5.36 & 8.2E-08 \\
\hline rs9401928 & 6 & 127298394 & RSPO3 & G & A & 0.55 & -0.02 & $2.7 \mathrm{E}-14$ & 4.44 & $9.0 \mathrm{E}-06$ \\
\hline rs6999835 & 8 & 78948855 & PKIA & $\mathrm{T}$ & $\mathrm{C}$ & 0.63 & 0.01 & 5.1E-09 & 3.74 & $1.8 \mathrm{E}-04$ \\
\hline rs10512176 & 9 & 89252706 & ZССНС6 & $\mathrm{T}$ & $\mathrm{C}$ & 0.72 & -0.01 & $3.5 \mathrm{E}-11$ & -4.07 & $4.8 \mathrm{E}-05$ \\
\hline rs10764494 & 10 & 25058144 & $A R H G A P 21$ & $\mathrm{C}$ & A & 0.32 & -0.01 & 4.7E-10 & 3.84 & $1.2 \mathrm{E}-04$ \\
\hline rs76567987 & 12 & 31037655 & TSPAN11 & A & G & 0.84 & 0.02 & 8.4E-16 & 4.01 & $6.1 \mathrm{E}-05$ \\
\hline rs9534439 & 13 & 47192049 & LRCHI & $\mathrm{T}$ & $\mathrm{C}$ & 0.19 & 0.02 & $5.1 \mathrm{E}-14$ & 4.36 & $1.3 \mathrm{E}-05$ \\
\hline rs61985972 & 14 & 59550263 & $D A A M 1$ & A & G & 0.94 & 0.03 & $5.2 \mathrm{E}-11$ & 5.02 & 5.2E-07 \\
\hline
\end{tabular}

Abbreviations: BETA, beta coefficient; CHR, Chromosome; EA, effect allele; FREQ, allele frequency of effect allele; NEA, non-effect allele; SNP, single nucleotide polymorphism; $\mathrm{P}, \mathrm{P}$ values.

UKBB, UK biobank data; IGGC, International Glaucoma Genetic Consortium; META, meta-analysis results of IGGC and EPIC-Norfolk datasets.

Chromosomal position is based on the NCBI RefSeq hg19 human genome reference assembly.

${ }^{1}$ A sample size weighted meta-analysis was conducted. 